\title{
Methicillin-resistant Staphylococcus aureus isolated from nonhealing wound in a canary bird (Serinus canaria domestica)
}

\author{
Zinka MAKSIMOVIĆ ${ }^{1, a}$, Alan MAKSIMOVIĆ ${ }^{2, b}$, Ismar LUTVIKADIĆ ${ }^{2, c}$, \\ Maid RIFATBEGOVIĆ ${ }^{1, d, \bowtie}$ \\ ${ }^{1}$ University of Sarajevo, Veterinary Faculty, Department of Microbiology and Infectious Diseases, Sarajevo, Bosnia and \\ Herzegovina; ${ }^{2}$ University of Sarajevo, Veterinary Faculty, Department of Veterinary Surgery, Sarajevo, Bosnia and \\ Herzegovina. \\ aORCID: 0000-0002-5988-8337; ' ${ }^{\mathrm{O} O R C I D: ~ 0000-0002-4127-9445 ; ~ ' ~}{ }^{\mathrm{ORCID}}$ : 0000-0002-3768-3007; \\ dORCID: 0000-0001-8948-618X. \\ ${ }^{\square}$ Corresponding author: maid.rifatbegovic@vfs.unsa.ba \\ Received date: 08.12.2020 - Accepted date: 01.07.2021
}

\begin{abstract}
Little is known about the presence of methicillin-resistant Staphylococcus aureus (MRSA) in a canary bird (Serinus canaria domestica). Although MRSA in a canary bird was previously reported, to the best of our knowledge, this is the first full description of the isolation and antibiotic resistance pattern of MRSA in this pet bird. A swab was taken from the nonhealing wound on the lateral thigh of a four-year-old, caged, housed alone, male canary bird. After the identification of Staphylococcus aureus, the antibiotic susceptibility profile of the isolate was obtained by the disk diffusion test. According to the resistance to Oxacillin and Cefoxitin, the isolate was identified as MRSA. The $m e c A$ gene was confirmed by PCR. The bird was treated by offering drinking water medicated with an injectable enrofloxacin formulation at $200 \mathrm{mg} / \mathrm{L}$ over 10 days period. Two weeks after therapy, intensive contraction of the wound was observed with a size reduction. A week later, the complete epithelization of the wound defect was verified. In this study, we could not confirm the source of infection in a canary bird, but we believe that transmission was from wild birds when the cage was putting out in order to allow the bird to sunbathe or more likely via contact with the owner. The results of this study underline the necessity for further investigations on the epidemiological role of canary birds as potential reservoirs of MRSA.
\end{abstract}

Keywords: Antimicrobial resistance, canary bird, exotic birds, $m e c A$, pet birds.

Staphylococcus aureus (S. aureus) is a well-known pathogen in humans and many animal species causing a wide range of infections (5). It is mainly recognized as an important cause of morbidity and economic loss in production animals, but it is also very important in companion animals $(13,15)$. Pet birds, including canary birds (Serinus canaria domestica), are common pets, and they can be infected with $S$. aureus in different forms, including pneumonia, air sac inflammation, pericarditis, and diarrhea. Moreover, S. aureus can cause death in canaries kept in aviaries (5). The most important zoonotic diseases associated with birds include chlamydophilosis, salmonellosis, tuberculosis, and highly pathogenic avian influenza (2). Numerous other bacteria also have zoonotic potential, such as Campylobacter spp, Borrelia burgdorferi, Pasteurella spp, Klebsiella spp, Yersinia spp, Pseudomonas spp, and Escherichia coli $(2,6)$. In recent decades, methicillin resistance of $S$. aureus has become an emerging problem in human and veterinary medicine (1). After the first isolation of methicillin resistant $S$. aureus (MRSA) from the milk of mastitic cow in 1972, MRSA has been increasingly found in many animals (8). The evidence that transfers of methicillin resistant $S$. aureus strains can occur between animals and humans $(8,11)$ increase the zoonotic potential of different animals as a source of MRSA infection in humans. Due to their close contact with humans, MRSA in household pets has potentially significant implications for human health, especially because of evidence of MRSA colonization in clinically normal animals (20). Despite the importance of MRSA in pets, there is very limited information about MRSA in exotic birds. So far, a few cases in parrots have been described $(3,14,19)$ and only one report of MRSA in canary birds, without any detailed case description (9). 
The purpose of this report is to describe isolation of MRSA from an unhealing wound in a household canary bird (Serinus canaria domestica).

A four-year-old, caged, housed alone, male canary bird (Serinus canaria domestica) was referred to the Surgery Department of Veterinary Faculty University of Sarajevo with a history of the nonhealing wound of unknown etiology, progressing in size despite undertaken, by veterinarian prescribed, topical therapy. The previous therapy consisted of wound irrigation twice daily for seven days with the $0.1 \%$ povidone-iodine antiseptic solution. Physical examination revealed a full-thickness wound in the right lateral thigh, approximately 7 to $10 \mathrm{~mm}$ in diameter, presented with some portions of the granulation tissue, exudate at the wound surface, and the redness of the surrounding skin. A wound swab was taken and immediately transfer to the laboratory for bacteriological examination. The swab was inoculated onto blood agar, MacConkey's, and bromocresol purple lactose agar and incubated aerobically for 24 hours at $37^{\circ} \mathrm{C}$. Preliminary identification of the isolate was performed by colony morphology, hemolysis, Gram stain, catalase test, tube coagulase test, and fermentation of maltose on bromocresol purple maltose agar (10). The final identification of $S$. aureus was done by polymerase chain reaction (PCR) using oligonucleotide primers described by Sasaki et al. (17).

Antimicrobial susceptibility testing of MRSA isolate was performed by a disc diffusion method following the guidelines of the Clinical Laboratory Standards Institute (4). The following antimicrobial disks (Conda) were used: Amoxicillin $(25 \mu \mathrm{g})$, Amoxicillin-Clavunalic acid (20/10 $\mu \mathrm{g})$, Ampicillin-Sulbactam $(10 / 10 \mu \mathrm{g})$, Cefoxitin $(30 \mu \mathrm{g})$ Chloramphenicol $(30 \mu \mathrm{g})$, Enrofloxacin $(5 \mu \mathrm{g})$, Clindamycin $(2 \mu \mathrm{g})$, Erythromycin $(15 \mu \mathrm{g})$, Gentamycin $(10 \mu \mathrm{g})$, Oxacillin $(1 \mu \mathrm{g})$ and Penicillin G (1 IU). For detection of mecA and mecC (mecA $\left.A_{\mathrm{LGA} 251}\right)$ (methicillin resistance) genes in the isolate, PCR was performed as described previously (18). Once the antibiogram results were obtained, topical treatment with $0.1 \%$ povidoneiodine antiseptic solution was discontinued, and oral application with enrofloxacin, was applied by offering drinking water medicated with an injectable enrofloxacin formulation at $200 \mathrm{mg} / \mathrm{L}$ over 10 days period. Written informed consent was obtained from the owner for all procedures undertaken.

According to the results of preliminary identification, the isolate was identified as coagulase positive staphylococci. S. aureus was finally identified by PCR. The S. aureus isolate was found to be resistant to Penicillin G, Amoxicillin, Amoxicillin-Clavunalic acid, Ampicillin-Sulbactam, Cefoxitin, Erythromycin, and Oxacillin. The isolate was intermediately susceptible to Clindamycin and susceptible to Chloramphenicol,
Enrofloxacin, and Gentamycin. Due to resistance to Oxacillin and Cefoxitin, the isolate was identified as MRSA. The presence of the $m e c A$ gene was confirmed by PCR.

After the $4^{\text {th }}$ day of antibiotic therapy, wound healing was evident by the absence of the exudate at the wound surface, a visible proliferation of the granulation tissue over the wound defect, and reduction of the redness of the surrounding skin. Two weeks after systemic antibiotic therapy was introduced, intensive contraction of the wound was observed with a concentric reduction in size. A week later, the complete epithelization of the wound defect was verified.

MRSA is well known emerged pathogen in human medicine since 1970s and has been increasingly reported as an emerging problem in veterinary medicine during the past 20 years (8). The prevalence of MRSA isolates in humans varies markedly between geographic areas. In veterinary medicine, distribution also depends on animal species. In recent years, MRSA has been identified in various animals, mainly in horses, food-producing animals, and increasingly in companion animals $(8,20)$. According to the European Food Safety Authority, the greatest risk to human infections are pigs, veal calves, and broilers. There are still no specific studies that examined the risk of human infection or colonization from small animals (1). However, the importance of small animals as a possible source of zoonotic infections in humans has substantially increased in proportion to the importance and number of pets in the life of a modern man. In the last two decades, isolation of MRSA has been increasingly reported from different household pets, mainly in dogs and cats, as well as in some exotic pets such as hamsters, rabbits, turtle, and bat $(11,14,19)$. Unlike other pets, MRSA findings in household pet birds are less common. There are only a few reports that confirm the presence of MRSA in parrots $(14,19)$, with only one full clinical description (3). Recently, Loncaric et al. (9) have reported the genetic diversity of MRSA strains isolated from companion animals in Austria, including one strain isolated from a canary bird. However, to the authors' knowledge, this study is the first full description of the isolation and antibiotic resistance pattern of MRSA from the household canary bird. Unfortunately, in this study we could not confirm the source of infection in the canary bird; however, it could be via contact with the owners, or with the wild birds. Namely, putting the cage outside in order to allow the bird to sunbathe, could be a condition favoring contact between pet and wild birds (2). Among wild passerines, MRSA has been detected in several species, including chaffinch (Fringilla coelebs) (12), spotted flycatcher (Muscicapa striata), robin (Erithacus rubecula) and common blackbird (Turdus merula) (7). Transfer of MRSA strains between animals and humans 
and vice versa has been reported several times $(8,16)$, so the infection from the humans to the canary bird in this study can not be excluded. A recent study by Loncaric et al. (9) has demonstrated that the majority of MRSA isolates recovered from small companion animals are human-related, which supports our theory that the isolate of MRSA in this study could be of human origin.

The results of this study strongly imply that a canary bird can carry MRSA and underline the need for further investigations on the epidemiological role of pet birds as potential reservoirs of MRSA. Also, this research confirms the importance of antibiogram testing in the prevention of antibiotic resistance in bacteria of animal origin. Furthermore, this is the first report of MRSA isolated from animals in Bosnia and Herzegovina.

\section{Financial Support}

This research received no grant from any funding agency/sector.

\section{Ethical Statement}

This study does not present any ethical concerns.

\section{Conflict of Interest}

The authors declared that there is no conflict of interest.

\section{References}

1. Andreoletti O, Budka H, Buncic S, et al (2009): Assessment of the Public Health significance of meticillin resistant Staphylococcus aureus (MRSA) in animals and foods Scientific Opinion of the Panel on Biological Hazards. EFSA J, 993, 1-73.

2. Boseret G, Losson B, Mainil JG, et al (2013): Zoonoses in pet birds: Review and perspectives. Vet Res, 44.

3. Briscoe JA, Morris DO, Rankin SC, et al (2008): Methicillin-resistant Staphylococcus aureus-associated dermatitis in a Congo African grey parrot (Psittacus erithacus erithacus). J Avian Med Surg, 22, 336-343.

4. CLSI (2012): Performance Standards for Antimicrobial Susceptibility Testing; Twenty-Second Informational Supplement. CLSI document M100-S22. Wayne, PA: Clinical and Laboratory Standards Institute.

5. Devriese LA, De Herdt P, Desmidt M, et al (1994): Pathogenic staphylococci and staphylococcal infections in canaries. Avian Pathol, 23,159-162.

6. Evans EE (2011): Zoonotic Diseases of Common Pet Birds: Psittacine, Passerine, and Columbiform Species. Vet Clin Exot Anim, 14, 457-476.
7. Kutkowska J, Turska-Szewczuk A, Kucharczyk M et al (2019): Methicillin-resistant Staphylococcus aureus and glycopeptide-resistant enterococci in fecal samples of birds from South-Eastern Poland. BMC Vet Res, 15, 1-8.

8. Leonard FC, Markey BK (2008): Meticillin-resistant Staphylococcus aureus in animals: A review. Vet J, 175, 2736.

9. Loncaric I, Lepuschitz S, Ruppitsch W et al (2019): Increased genetic diversity of methicillin-resistant Staphylococcus aureus (MRSA) isolated from companion animals. Vet Microbiol, 235,118-126.

10. Markey B, Leonard F, Archambault $M$ et al (2013): Staphylococcus species. 105-115. In: Clinical Veterinary Microbiology. Mosby Elsevier.

11. Morgan M (2008): Methicillin-resistant Staphylococcus aureus and animals: Zoonosis or humanosis? J Antimicrob Chemother, 62, 1181-1187.

12. Paterson GK, Larsen AR, Robb A et al (2012): The newly described mecA homologue, mecALGA251, is present in methicillin-resistant Staphylococcus aureus isolates from a diverse range of host species. J Antimicrob Chemother, 67, 2809-2813.

13. Pomba C, Rantala M, Greko C et al (2017): Public health risk of antimicrobial resistance transfer from companion animals. J Antimicrob Chemother, 72, 957-968.

14. Rankin S, Roberts S, O'Shea K et al (2005): Panton valentine leukocidin (PVL) toxin positive MRSA strains isolated from companion animals. Vet Microbiol, 108, 145148.

15. Rubin JE, Ball KR, Chirino-Trejo M (2011): Antimicrobial susceptibility of Staphylococcus aureus and Staphylococcus pseudintermedius isolated from various animals. Can Vet J, 52, 153-157.

16. Rutland EB, Weese JS, Bolin C et al (2009): Human-todog transmission of methicillin-resistant Staphylococcus aureus. Emerg Infect Dis, 15, 1328-1330.

17. Sasaki T, Tsubakishita S, Tanaka Y et al (2010): Multiplex-PCR method for species identification of coagulase-positive staphylococci. J Clin Microbiol, 48, 765-769.

18. Stegger M, Andersen PS, Kearns A et al (2012): Rapid detection, differentiation and typing of methicillin-resistant Staphylococcus aureus harbouring either mecA or the new mecA homologue mecALGA251. Clin Microbiol Infect, 18, 395-400.

19. Walther B, Wieler LH, Friedrich AW et al (2008): Methicillin-resistant Staphylococcus aureus (MRSA) isolated from small and exotic animals at a university hospital during routine microbiological examinations. Vet Microbiol, 127, 171-178.

20. Weese JS, van Duijkeren E (2010): Methicillin-resistant Staphylococcus aureus and Staphylococcus pseudintermedius in veterinary medicine. Vet Microbiol, 140, 418-429. 